\title{
Successful Friendships of Hispanic Children and Youth With Disabilities: An Exploratory Study
}

\author{
Ann P. Turnbull, Martha Blue-Banning, and Lourdes Pereira
}

\begin{abstract}
Interviews were conducted with Hispanic children who had a disability and a friend with whom they had a successful friendship, as well as with parents and teachers. We focused the interviews on the children's and adults' descriptions of the friendship, what each of the children gave to and received from the friendship, the evolution of the friendship in terms of intensity, and the influence of Hispanic cultural values. A friendship support conceptual framework is presented to organize the data related to three relationship domains (companionship, instrumental support, and emotional support) and three friendship depth levels (acquaintance, casual, and intimate). We focused our key recommendations on future directions for research and friendship
\end{abstract}

A focus on friendship has been a burgeoning area of interest within the special education field over the last several years. A recent emphasis has been on studying the nature of friendship interactions between children with and without disabilities (Farmer \& Farmer, 1996; Helmstetter, Peck, \& Giangreco, 1994; GrenotScheyer, 1994; Meyer, Park, GrenotScheyer, Schwartz, \& Harry, 1998; Salisbury, Gallucci, Palombaro, \& Peck, 1995; Snell \& Vogtle, 1997; Staub, 1998; Staub, Schwartz, Gallucci, \& Peck, 1994).

Although there is a rich literature related to friendships and social support in typical child, youth, and adult development (Belle, 1989; Sarason, Sarason, \& Pierce, 1990), much of the work in the disability field has not been grounded in that literature. Children without disabilities gradually increase their contact with peers as they grow older, with a $70 \%$ increase between the ages of 6 and 9 . By this time, typical children usually have 9 or 10 friends in their social network (Feiring \& Lewis, 1989). Although theorists and researchers have categorized social relationships of typical children and youth in different ways, frequently identified re- lationship domains are emotional support, informational support, instrumental support, companionship, and affection (Belle, 1989; Berndt, 1989; Reid, Landesman, Treder, \& Jaccard, 1989; Sullivan, 1953). Similar relationship domains have been identified in the adult literature on social support (Cobb, 1979; Cutrona \& Russell, 1990; Schaefer, Coyne, \& Lazaruz, 1981).

A tendency within special education settings is for helping relationships to be characterized automatically as friendships. Helping relationships (i.e., peer tutoring, behavioral monitoring) fall within the relationship domains of instrumental support and/or informational support. Evans, Salisbury, Palombaro, Berryman, and Hollowood (1992) documented that girls, more frequently than boys, interact with students with severe disabilities by serving as a personal attendant or caretaker in terms of moving students around the room, getting materials for them, and making sure that their special needs are met. Peers often comment that they "work with" a classmate who has a disability (Kishi \& Meyer, 1994). Distinguishing between working with someone and experiencing friendship is critically impor- 
tant, as described by Van der Klift and Kunc (1994):

Clearly, there is nothing wrong with help; friends often help each other. However, it is essential to acknowledge that help is not and can never be the basis of friendship.... Friendship is about choice and chemistry and cannot even be readily defined, much less forced. (pp. 393-394)

Perhaps, a contributing factor to an overreliance on a helping model has been the limited attention of researchers in the special education field on the explication and facilitation of reciprocity (Grenot-Scheyer, Staub, Peck, \& Schwartz, 1998; Kennedy, Horner, \& Newton, 1990; Kennedy \& Itkonen, 1996; Salisbury \& Palombaro, 1998). Alternatively, in the social support literature for typical children, youth, and adults, investigators have emphasized the importance of establishing reciprocity balance within relationships (Antonucci \& Jackson, 1990; Ross, Cheyne, \& Lollis, 1988; Sabatelli \& Shehan, 1993).

In addition to considering the nature of relationship domains and the reciprocity balance between giving and receiving support within domains, researchers in the area of social supports for typical children, youth, and adults also address relationship depth. Hays (1984) described three levels of relationship depth, including superficial, casual, and intimate. In his research he documented the evolution of college students' dyadic relationships that result in intimate friendships and those that do not result in intimate friendships. Of his many findings related to the evolution of intimate friendships, the most striking behavioral difference between the dyads who established an intimate friendship, as compared to those who did not, was the quantity of interaction within relationship domains occurring between 3 to 6 weeks of acquaintanceship.

Grounded in the social support literature for typical children, youth, and adults in terms of relationship domains and levels of depth, we undertook a qualitative study to inquire into the nature of friendships between 11 Hispanic children with disabilities and their peers without disabilities. (The term Hispanic generally refers to individuals whose cultural heritage traces back to a Spanish-speaking country with a history of Spanish-American colonization [Harry, 1992]. Many other labels have been used to denote this population, such as Latino, Spanish, and Latin. Currently, there is no consensus among social scientists as to the most acceptable term to use [Marín \& Marín, 1991; Soriano, 1991]. In the early 1970s, the federal government adopted the term Hispanic, which has subsequently been designated as the official term for use by the U.S. Bureau of the Census and in all federal publications [Gallegos, 1991; Walker, 1987]. For consistency, Hispanic is the term used throughout this article.) In initially planning this study, our intention had been to include participants from the three racial/ethnic groups of Hispanic, African American, and EuroAmerican. In discussing participant selection with our Participatory Action Research Committee (described in the next section), we were strongly encouraged, particularly by two members of this committee who are not Euro-American, to focus solely on one cultural group rather than three. Their rationale was that they believed it would be important to understand more about friendships from specific cultural traditions. Their recommendation was to focus on Hispanic children and youth. We concurred with this recommendation concerning cultural depth for several reasons. First, the Hispanic population is one of the fastest growing racial/ ethnic groups in the United States (Gutiérrez, 1995). In fact, it is projected to be the largest culturally diverse population by 2010 , comprising approximately $24 \%$ of the total U.S. population (U.S. Bureau of the Census, 1995). Second, much of what we know in the disability field is known primarily from a Euro-American perspective. Although we have not conducted a "cultural analysis" of recent research on social relationships, we have analyzed the ethnic composition of participants in research published on family topics during 1998 in the early childhood field in four leading journals (Journal of Early Intervention, Topics in Early Childhood Special Education, InfantToddler Intervention: The Transdisciplinary Journal, and Infants and Young Children). Of the 11 empirical articles that had the terms parent, family, mother, father, or primary caregiver in the title, Hispanic family members were represented in only $5 \%$ of the research samples. This is in contrast to the fact that Hispanic students represent $12 \%$ of the total population and $28 \%$ of the population of students identified as having mental retardation (combining mild and moderate identification) (U.S. Department of Education, 1993). Third, we chose to focus on Hispanic children and youth because of the emphasis on positive interpersonal relationships in the Hispanic culture. For example, a strong Hispanic cultural value is personalism, which places great importance on interpersonal relationships (Soriano, 
1991), and simpatíca, which emphasizes harmony in interpersonal relationships (Marín \& Marín, 1991). In light of these Hispanic cultural values, we anticipated that there may be lessons to learn from successful Hispanic friendships that would be helpful for people with diverse cultural values who may be less inclined to give high priority to interpersonal relationships. In providing this rationale, we want to point out, however, that we recognize that there is extreme variability within the Hispanic population in light of many factors, including national origin, level of acculturation, language proficiency, and socioeconomic status.

The four research questions addressed in this paper are: (a) What do children with and those without a disability who experience a successful friendship do to give support to each other and to receive support from each other? (b) To what extent is there reciprocity in the ways that children/youth with and those without a disability give support to each other and receive support from each other? (c) How is friendship depth among children with and those without a disability characterized? (d) In what ways do Hispanic cultural values influence the friendships of children with and those without a disability?

\section{Method}

In initially undertaking this study, we established a Participatory Action Research Committee to advise us in the specification of relevant questions and the implementation of research to increase the likelihood that the intended beneficiaries of the research-children with and those without a disability, parents, teachers, and researchers-would, indeed, gain from the findings (Hoshmand \& Polkinghorne, 1992; Lather, 1986; Meyer et al., 1998; Turnbull, Friesen, \& Ramfrez, 1998). This committee was comprised of one adult with a disability, four parents, two teachers, and two researchers.

We used individual and group interviews for data collection. In-depth interviews enabled inductive inquiry that allowed us to generate recommendations for future research and intervention on friendship facilitation.

\section{Participants}

Given that our goal was to take a strengths perspective (Saleebey, 1992) to enhance our understanding of the successful friendships of Hispanic children with mild, moderate, or severe disabilities, we used purposive and extreme case sampling to select participants whose friendships met specified criteria associated with success. The purposive sampling (the selection of a reduced number of respondents to represent the target group's diverse characteristics [Bogdan \& Biklen, 1998; Lincoln \& Guba, 1995]) involved seeking children characterized by diversity across geographical residence, age, type of disability, and nature of disability. The extreme case sampling (choosing participants who demonstrate special or unusual situations that might be especially instructive [Patton, 1990]) involved the selection of children with a disability who were known to have a successful friendship with a peer who did not have a disability. Although there are alternative interpretations of what constitutes a successful friendship, we set the following criteria as the basis for identifying children based on a review of the friendship and social support literature related to children without disabilities (Belle, 1989): (a) the two children's ages are within 18 months of each other; (b) they both initiate contact and activities with each other; (c) they share experiences in at least two settings (i.e., school playground and neighborhood); and (d) they have known each other for a minimum of 6 months.

Given the exploratory nature of this study, we selected 11 Hispanic children who represented variation in age (i.e., 6 to 19), disability classification (i.e., mental retardation, autism, and emotional/behavioral disorders), geographic location (i.e., rural, suburban, and urban), and Hispanic subgroup classification (i.e., Mexican and Puerto Rican). In terms of Hispanic subgroup classification, 9 of the children and youth were Mexican and 2 were Puerto Rican. We selected 12 friends (one youth with a disability had 2 close friends who were equally the focus of interviews).

Because of our interest in varied geographic locations, we established four community research sites in (a) Texas, (b) California, (c) Kansas, and (d) Connecticut. Given the distance and money required for travel to these sites, we determined that it would be feasible for us to go only one time and, thus, have one interview with each participant. Rather than being able to arrange for repeated interviews, we sought to provide a thick description (Maxwell, 1996) of each child's friendship by interviewing the child with a disability and the parent(s) of the child with a disability in all circumstances. When possible, we also interviewed the siblings of the person with disability, parent(s) of the friend without a disability, a teacher of both the child with a disability and the friend without a 
disability. Table 1 identifies the 56 respondents-children, parents, siblings, and teacherswho participated in this study.

Locating participants was a major challenge. One of the specific aspects that made it challenging was that many children with disabilities were reported not to have successful friendships. In addition to this challenge, finding eligible individuals also involved a lengthy process of participant identification, given that we had not previously worked in the communities and needed to establish a trusting relationship with at least one Hispanic community leader as an entry strategy into the community in order to eventually identify study participants.

We identified a Hispanic community leader most were indigenous parent leaders) within each of the four targeted communities) to serve as a

Table 1

Characteristics of Respondents

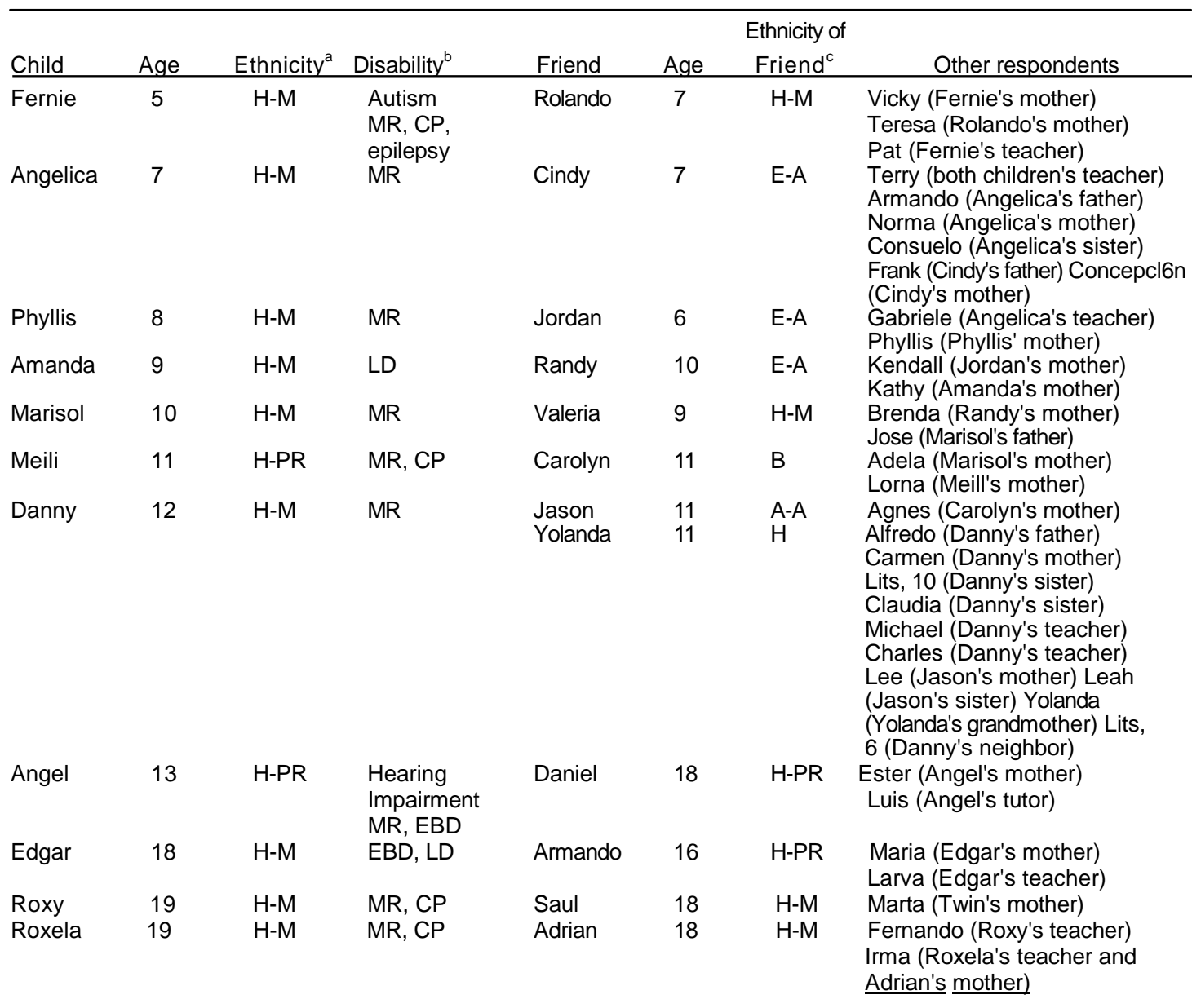

${ }^{\mathrm{a}} \mathrm{H}-\mathrm{M}=$ Hispanic (Mexican); H-PR = Hispanic (Puerto Rican); E-A = Euro-American; $\mathrm{A}-\mathrm{A}=$ African American; $\mathrm{B}=\mathrm{Black}$ (Caribbean). ${ }^{\mathrm{b}} \mathrm{CP}=$ cerebral palsy, $\mathrm{MR}=$ mental retardation, $\mathrm{LD}=$ learning disability, $\mathrm{EBD}=$ emotional and behavioral disorders. ${ }^{c}$ Not Interviewed. 
research site coordinator. These site coordinators assisted us in (a) identifying and prioritizing Hispanic children with disabilities who met the study's friendship criteria; (b) contacting the children and their parents to explain the purpose of the study and obtain their consent; (c) assisting the parents in inviting their child's teacher and their child's friend who did not have a disability to participate; (d) contacting the teacher, the friend without a disability, and the parents of the friend to explain the study and ask them to participate; (e) making logistical arrangements for a comfortable and convenient location for the interviews; (f) scheduling the interviews; (g) arranging for transportation, child care, and refreshments; (h) facilitating the completion of consent and demographic forms; and (i) being present at the interviews to reassure the children, parents, and teachers and to handle any unexpected issues. Each site coordinator was paid $\$ 150$ to carry out this role.

Because the site coordinators were actively involved in the Hispanic community, they knew many families across different neighborhoods and schools who had children with disabilities. They were able to recommend families whose children met the criteria and to contact other families and professionals to attain their nominations. By networking with the site coordinators, we were able to engage in a reflective process that helped them prioritize the particular children with a disability and their friends who would be most appropriate to include from their geographical setting.

Based on a review of the friendship literature and the comments of the Participatory Action Research Committee, we developed a semistructured interview guide to provide general direction for the individual and group interviews (Creswell, 1994; Krueger, 1998). Consistent with the literature reviewed in the introduction, the interview guide included questions focused on relationship domains (i.e., emotional support, informational support, instrumental support, companionship, and affection), reciprocity within relationship domains, relationship depth, and the role of Hispanic culture in influencing friendships. We adapted this interview guide for the level of reading comprehension of each child as well as for the adults. We also adapted it in an iterative way, as issues were identified by participants in early interviews which helped shape questions for later interviews. Participants were also encouraged to identify and elaborate upon factors and barriers that influence the development and maintainence of friendships. Although these topics provided the overall paramaters for the interviews, the participants determined the sequence and course of the conversations in terms of issues that were most important to them. They were given the choice of having the interview in English or Spanish; 9 of the 56 respondents chose Spanish. Four interviewers conducted the interviews, one of whom was fluent in Spanish. Participants selected whether they would prefer to have the interviews individually or in a group. In most situations, the child with a disability and the friend had the interview together (they typically reported feeling more comfortable being interviewed together rather than individually), whereas parents and teachers typically had separate interviews. All interviews were audio-recorded and fully transcribed. The interviews were designed to provide an informal exchange (Taylor \& Bogdan, 1998, p. 77). The support of the site coordinator gave reassurance to families who were meeting us for the first time. The children received a \$5.00 McDonald's coupon; in two of the sites, the adults received a $\$ 10.00$ check; in one site, a $\$ 15.00$ grocery coupon; and

in another site, a $\$ 25.00$ grocery coupon. (The site coordinators in each community recommended the appropriate renumeration.) In addition, all participants received a free subscription to a family and disability newsletter and a copy of the study's results.

\section{Data Analysis}

The goals of data analysis were to (a) organize and reduce the raw data; (b) generate categories, subcategories, and codes; and (c) interpret patterns and themes. In terms of organizing and reducing the raw data, the transcripts generated 32,088 lines of text that were divided into 29 categories. Two researchers read and re-read all transcripts to become thoroughly familiar with the data and to mark relevant passages. These passages were organized according to themes and provided the basis for category generation (Berg, 1998; Merriam, 1998). A third researcher read a portion of the transcripts for the purpose of confirming credibility (discussed in the next section).

In the second step we generated categories, subcategories, and codes by (a) developing a preliminary list of categories, (b) discussing and refining the similarities and differences between 
categories, (c) designating a code consistent with each category, and (d) locating passage categories within the transcript and marking them with the designated code. A research assistant entered all data using Ethnograph, a computer software program that is a qualitative data management tool (Seidel, Friese, \& Leonard, 1995). The first and second authors (a) reviewed the categories as sorted by Ethnograph and (b) specified subcategories using the same process as the category determination.

Finally, the team interpreted patterns and themes by (a) discussing the meaning of each category and subcategory and seeking the consistencies, inconsistencies, and key quotes associated with each; (b) searching for possible explanations and interpretations; (c) tying possible explanations to the literature; (d) sharing the findings with participants (this is the member check, which will be discussed later) and the Participatory Action Research Committee (this is the stakeholder check, which will also be discussed later), and (e) clarifying interpretations through the process of writing the results.

We ensured credibility (accuracy of the information) by using multiple respondents, multiple researchers, and member/stakeholder checks. The number of multiple respondents for each friendship set ranged from 4 to 13 respondents. This provided a breadth of information that helped to counteract the logistical and fiscal impracticality of conducting repeated interviews with the same respondent in four diverse geographical settings. Multiple researchers provided a system of checks and balances for data collection by classifying the passages into categories/subcategories and coding the transcripts.

We conducted a member check by sending a copy of this article and a feedback questionnaire to 4 parents and 1 youth- 2 respondents from one research site and 1 respondent from each of the other three sites. The 2 respondents indicated that they did not disagree with anything in the article, and they did not believe that important points were left out. The stakeholder check involved sending a copy of the paper to the nine members of the Participatory Action Research Committee, with a specific request for feedback from five of them. The respondents approved the content of the article and requested further details, especially in terms of disability identification (disability identification was subsequently added to Table 1).

\section{Limitations of the Study}

In this study we explored the nature of support that children with and those without a disability provide to each other through their friendship experiences, the extent of reciprocity, the characterization of friendship depth, and the impact of Hispanic cultural values. Given the exploratory nature of research on friendships between children with and those without a disability, the use of qualitative inquiry is particularly appropriate in providing a thick description of data related to the research questions. As is the case with qualitative inquiry, generalizations from these findings to all Hispanic families or to all families, regardless of culture, are highly inappropriate to make.

For data collection, we relied on a one-time interview. Although we would have preferred to repeat interviews, the funding for our study made such extensive travel prohibitive. We chose to collect information from participants in four locations in the United States rather than to select families within driving distance whom we could interview repeatedly. We attempted to compensate for this lack of depth in repeated interviews by having breadth in interviews in terms of getting the perspectives of a number of different people (i.e., child with and without a disability, parents, siblings, teachers) related to each of the friendships.

Another limitation was the difficulty we experienced in defining friendship and in being confident about exactly what constitutes a successful friendship. Although we relied on the social relationships literature related to children and youth without disabilities (Belle, 1989) in specifying criteria, we believe that future researchers need to operationalize definitions and validate quality indicators related to successful friendship within and across diverse cultural contexts.

An unanticipated consequence is that the present study includes friendship sets that did not meet our original friendship criteria related to (a) the friend not having a disability and (b) the age difference being within 18 months, as we discuss later in this section. Interestingly, some of those friendships that did not meet our original criteria represent some of the more intimate friendships. As we discuss in the next section, we think the "violation" of this study's participant criteria worked to our advantage because it 
caused us to consider the limitations of our own beliefs about the criteria for successful friendships.

Another limitation was that we did not collect demographic information on the severity of each child's disability. We regret that we cannot more definitively describe the nature and extent of disability, although we noted quite similar friendship issues when we compared the child with the least severe disability with the child with the most severe disability. We recommend that future researchers definitively clarify the impact of the nature of the disability on relationship domains and friendship depth.

Likewise, we did not collect adequate information on the family's socioeconomic level or level of acculturation. We acknowledge that these are critically important variables to explore in relation to successful friendships, and we recommend that future researchers take these variables into account.

Our original intention was to have approximately one half of the Hispanic children with disabilities from Mexico as their country of origin, one fourth from Puerto Rico, and one fourth from Cuba; however, we were unsuccessful in obtaining this degree of Hispanic diversity. We had originally planned to obtain a Cuban population in the Miami area, but fiscal and logistical barriers prevented that from occurring. We also were not able to locate as many Puerto Rican children in the Hartford area as we had hoped. The result was that the overwhelming majority of participants are from Mexico. Thus, we are unable to make any speculations about various subgroups of Hispanic populations in regard to successful friendships.

Although we conducted some interviews in Spanish, transcribed the interviews in Spanish, and then translated them into English, we did not re-translate these transcripts to assure reliability in translation. The translator, however, had extensive experience in translations and consistently received highly favorable feedback on the quality of her translations.

A final methodological limitation is that we did not have a professional peer conduct a confirmatory analysis of our analytic process and procedures; however, multiple researchers coded the data, and we conducted member and stakeholder checks. In light of the exploratory nature and the aforemented limitations of this study, we highlight findings in the next section.

\section{FINDINGS}

In presenting the findings, we integrate the data on relationship domains and friendship depth into the presentation of a friendship support conceptual framework. We then report data on the specific influence of Hispanic cultural values on friendships.

Figure 1 summarizes the findings of this study related to relationship domains and depth of friendship. We developed this conceptual framework after we analyzed the data as we were in the process of attempting to synthesize it. In addition to using the grounded data in developing the framework, we considered findings from the literature reviewed in our introductory section related to relationship domains and relationship depth. Although researchers have generally focused on five domains (i.e., emotional support, informational support, instrumental support, companionship, and affection) in the social relationship literature, the data from our study suggested extreme difficulty in distinguishing informational and instrumental support as well as emotional support and affection. Thus, in Figure 1, we included three rather than five relationship domains. The proportional sizes of the domains and of the giving and receiving components within each domain in Figure 1 present the proportional coding of

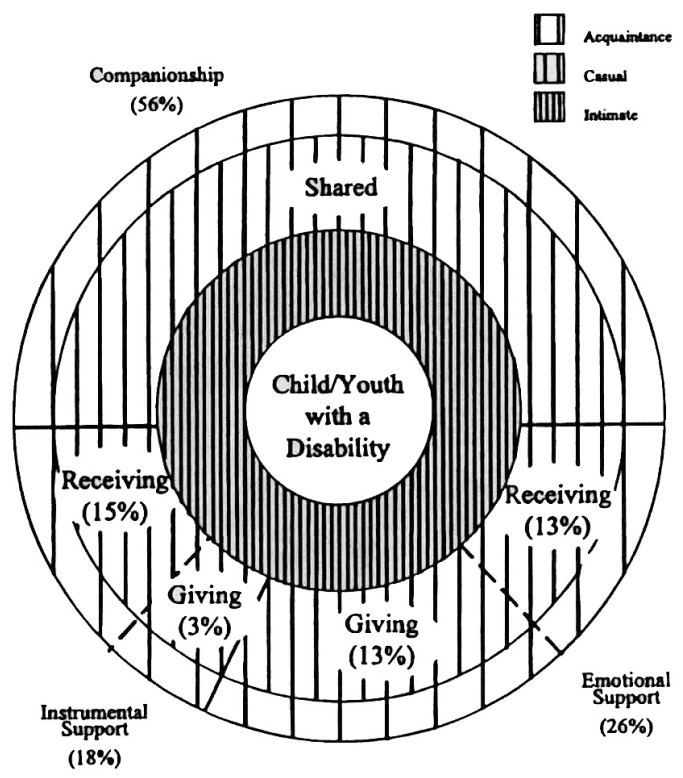

Figure 1. Friendship support conceptual framework.

respondents' comments that pertain to each domain to each aspect of reciprocity.

We were also intrigued by the research of Hays (1984), who described three levels of friendship depth; however, the concept of relationship depth typically has not characterized the friendship literature for children without disabil- 
ities. Although there are a number of discussions in the disability literature about the distinction between helping and friendship, there has not been a clear explication of the process in friendships evolving from an initial acquaintance to a more intimate level. Thus, Figure 1 illustrates the juxtaposition of relationship domains (i.e., instrumental support, emotional support, and companyionship), reciprocity (i.e., giving and receiving support within each domain), and relationship depth (i.e., acquaintance, casual, and intimate).

\section{Friendship Support Conceptual Framework}

We present the findings, consistent with Figure 1, by addressing (a) companionship, (b) instrumental support, (c) emotional support, and (d) depth of friendship. Within the relationship domains of instrumental and emotional support, we address the support that children with a disability give to and receive from friends. (The order of subcategories within each of the categories of companionship, instrumental support, and emotional support is consistent with the order of frequency with which participants describe them.

\section{Companionship}

We categorized the comments on companionship into the following six subcategories: (a) engaging in sports and physical activities, (b) visiting and talking, (c) going places, (d) playing, (e) engaging in artistic expression, and (f) participating in school and community activities. An example of a quote illustrating the companionship subcategory of going places is as follows:

I take her to the park ... with her friends, so she can have a good time. We stay half an hour or an hour until they get tired. Then, I take them to McDonald's on the way home. (parent of child with disability)

All friendships included at least three companionship subcategories.

Figure 1 illustrates that the companionship relationship domain received substantially more comments $(56 \%)$ than did the other two domains-approximately three times more than instrumental support and two times more than emotional support. There was a much more even distribution of comments across all 11 friendship sets in this category as contrasted to the instrumental and emotional support categories. In the relationship domains of instrumental support and emotional support, it was obvious from comments that one of the children in the friendship dyad was giving support and the other was receiving it. In contrast, the comments on companionship could not be analyzed in terms of the giving and receiving of support; rather, the emphasis was on having fun together.

\section{Instrumental Support}

In terms of instrumental support, $18 \%$ of the comments could be classified into this area, with $15 \%$ of all comments focusing on the instrumental support that the child with a disability receives from his or her friend and $3 \%$ of the comments focusing on the instrumental support that the child with a disability gives to the friend (see Figure 1). Approximately one third of the instrumental support comments focused on one adolescent, indicating a very substantial amount of instrumental support that he received from his friends without a disability.

Instrumental support received by child with disability from friend. We categorized the comments on instrumental support received by the child with a disability into four subcategories: (a) providing information, (b) providing assistance with school tasks, (c) providing practical help, and (d) providing advocacy.

Providing information. The most frequently mentioned subcategory was providing information. Comments indicated that the majority of friends without a disability provided information to the child with a disability on (a) what to do (i.e., eat chili chips, take books to class, state preferences, and wait for turn), and (b) how to do things (i.e., play basketball, swim, ride a bike, tie a shoelace). The following passage provides an example of providing information when suggestions were made about both what to do and how to do it:

We be walking and he see a girl by herself, and I be like why don't you go talk to her. An he be like no. Sometimes he tell I don't know what to say, and I'll like just say the first thing that comes to your mind.... I'll be like well, you may never see her again, maybe she be with someone else. The way I see it in life you gotta take chances. You got to go with what you feel inside. (friend without disability)

Providing assistance with school tasks. Comments pertaining to approximately half of the students concerned assistance they received with school tasks from their friend. Examples of school tasks with which they received assistance included reading, math, writing, paying attention in class, and completing homework. There was not a strong emphasis, however, on the role of friends being tutors. The academic help was described as 
being more incidental and intermittent, as contrasted to regularly scheduled tutoring.

Providing practical help. This subcategory involved the friend actually doing something for the child/youth with a disability, and comments indicated that the majority of friends provided practical help. The practical help themes included (a) assistance with orientation to school building and with mobility, (b) assistance with social interaction, and (c) general assistance.

Providing advocacy. Providing advocacy refers to friends standing up for the children/youth with a disability in helping to defend their rights for respect and participation in the school. Only one student with a disability, Danny, received this type of assistance; he received a substantial amount as indicated by the pervasive way in which providing advocacy was addressed in all related interviews. Interestingly, Danny was the only student with a circle of friends, and his advocacy appeared to be directly related to the circle. As illustrated by the following quote:

When we were in fifth grade ... the principal, she didn't give Danny the rights that other kids had and one day when we met here for the meeting we all decided to go and stand-up for Danny. So we went to Miss [principal] and we told her that, 'Why can't Danny have the rights that other kids are getting?' (friend without disability)

Instrumental support given by child with disability to friend. Only $3 \%$ of the comments related to instrumental support were ones in which the child with the disability was giving support to the friend. The two subcategories of instrumental support given by the child with a disability to the friend include (a) providing assistance with school tasks and (b) providing information. As contrasted to the four categories of instrumental support received by the child with a disability from the friend, children with a disability did not give support to their friend in the subcategories of providing practical help and providing advocacy.

Approximately half of the children with a disability made a comment related to the provision of instrumental support for friends. The most instrumental support was provided by the older students with a disability who were especially verbal.

Providing assistance with school tasks. This was the most frequent subcategory mentioned. Examples of school tasks include assistance with coloring within the lines, handwriting, and organizing extracurricular activities. Jordan's kind of slow.... He's in kindergarten, but he didn't go to preschool.... Jordan didn't stay in the lines good. She [Phyllis] said, "'Jordan, you're suppose to stay in the lines." (parent of friend without disability)

Providing information. All of the comments in this category related to Angel and Daniel's friendship. Angel was primarily providing Daniel information related to his hobbies.

\section{Emotional Support}

As illustrated in Figure 1, the emotional support category received approximately one fourth $(26 \%)$ of the comments related to the domains of friendship. Unlike instrumental support, children with a disability gave the same amount of emotional support to their friend $(13 \%)$ as they received from their friends (13\%).

Emotional Support Received by Child With Disability From Friend. Emotional support received by the child with a disability included the following three subcategories: (a) attending to feelings, (b) expressing affection and caring, and (c) enhancing self-esteem. As reflected in Figure 1 , the comments describing the amount of emotional support received by the child with a disability was smaller than the number of comments describing the amount of instrumental support received. The two children with disabilities to whom the most emotional support comments were attributed were both females, ages 7 and 9; furthermore, both of their friends were female.

Attending to feelings. Often this category related to comforting the child with a disability in terms of asking them about problems, reminding them of their friendships, and showing allegiance when they are teased. Sometimes it also involved being able to intuit feelings from nonverbal expressions when feelings were not directly verbally shared.

Expressing affection and caring. Friends expressed affection and caring in both physical and verbal ways. Comments related to affection occurred for 4 of the children with a disability. In three out of four of these situations, the individual with a disability was female.

Enhancing self-esteem. This category, related to interactions that help to increase positive feelings toward self, was the least frequently addressed. One of the few illustrative quotes is as follows: "He sees Fernie as equal to any child in that he can learn as any other child. In other words, he gives him support and trust" (parent of child with disability).

Emotional Support Given by Child With a Disability to Friend. Children with a disability 
gave emotional support to their friend in the same three subcategories in which they received it.

Attending to feelings. This category included comments related to providing comfort (i.e., asking about problems, showing allegiance when friend is teased) and understanding when the friend is in a bad mood (i.e., "Like when I'm in a bad mood or something [he] just comes and pats me on the back and says you're my best friend.")

Expressing affection and caring. Hugs, being nice, being tender, and having somebody to love were all described as ways of expressing affection and caring.

Enhancing self-esteem. There were more comments related to how children/youth with a disability enhance the self-esteem of their friends rather than vice versa. Several of these relate to how Angel provides a boost to Daniel's self-esteem.

\section{Depth of Friendship}

As depicted in Figure 1, our conceptual framework has three concentric circles representing three levels of depth-acquaintance, casual, and intimate. We would not characterize any of the friendships investigated in this study as being at the acquaintance level, which is not surprising given the criteria that we had for identifying successful friendship. Of the 11 friendship sets, we would classify 5 of them as casual and 6 as intimate. Although it is extremely difficult to establish operational criteria to separate these levels, we designated the intimate level when friendships were characterized by comments indicating (a) an intensity of emotion-al support and/or (b) overt affirmations of closeness. The following are three quotes illustrat-ing intimate friendships.

Since the first day I saw Meili, I really liked her. And I've been coming sometimes, and we really got to know each other and then now, its like we're family. (friend without disability)

A friend is someone who will always be there when you need them [He] will talk to you when you need help and when you don't need help. [He] will be there even when you've been-excuse my language-such an ass that nobody wants to be there, but your friend is there.... I love Angel like a brother. I love my other friends, but from all the friends that I have, he's been the only one that I can look at and say, "We did all this together, and we're going to stick together.... Because I know one day I might end up getting married and have my own life and stuff, but that doesn't mean I'll stop - if you're [Angel] still around I'll always work for you. No matter if Im a few miles away, I could be in Florida ... but I'm going to still take some time off and work for you and stuff. Because not only you're my friend, I feel you're part of my family.... you're always there, and I'm there for you and you're there for me. (friend without disability)

I know, like last year, I sat in on one of his Circle Friends, and it was incredible. Here are these elementary students having some serious heart-to-heart talks. (teacher)

In the friendships we identified as being at the casual level, comments suggesting this level of intimacy were not included.

\section{Influence of Hispanic Culture}

We asked all of the parents and teachers we interviewed to describe how the families' Hispanic culture influenced the nature of the friendship between the child with a disability and the friend without a disability, as well as the roles of the parents and teachers as friendship facilitators. Based on comments, we identified benefits and drawbacks. We want to acknowledge the broad diversity within the Hispanic culture and the impossibility of identifying these benefits and drawbacks to all Hispanic children and their families. We underscore, again, the inappropriateness of generalizations.

\section{Friendship Benefit of Hispanic Culture}

When specifically asked whether and how their Hispanic cultural values and traditions influenced their child's friendships, 4 of the parents of children with a disability strongly emphasized the broad network of social relationships provided by their relatives, in general, and cousins, in particular. Three additional families casually mentioned relatives, and 3 families did not mention the relatives as being a friendship resource (i.e., in one instance, the relatives were in Puerto Rico and the distance prevented them from playing an active role in the child's life).

Of the 4 families who discussed (with fervor) the relatives (especially cousins) as a rich reservoir of friendship support, there was a major theme of a "ready-made" social network for the child. Marisol's mother particularly emphasized the network of cousins:

I invite all her cousins and her friends who live on our same street... [my husband's] four brothers and sisters 
[and their children live in the same neighborhood].... All her cousins live across the street.

Marisol's social network, therefore, consisted primarily of her cousins, who are her friends as well. Her mother described how the roles of friends and cousins interact:

They have a beautiful relationship even though Marisol has some problems and Valeria is a normal kid. $A$ friendship beyond their relationship as cousins. When they are playing, they are just friends.

Angelica's father described "her little circle of cousins, they're basically the same age, they are growing up together." He explained that it was important for him to talk with the cousins about Angelica's special needs, so that they would feel more comfortable including her.

In addition to support from cousins, Phyllis' mother described how the broader network of relatives support:

I've got sisters-I've got a lot of sisters and brothers that whenever $I$ needed help, need baby-sitter, they are always there. They were always so positive about everything. They are more positive than $I$ am. She's got wonderful Godparents that are Hispanic that take her whenever $I$ need-my husband and $I$ need to get away. Our religion, we are Catholic. They say that Phyllis was a Godsend-our religion is real strong and so to them it's a little angel that was sent to us, and the whole family loves her, and my sisters have talked to their sons and daughters and they explained to them and to watch and take care of Phyllis. If you ever see anything or anybody mistreating, and some of them, like $I$ said are younger and they'll come and tell us if Phyllis is out of the yard or whatever. So they all know that Phyllis is special and they all take care of her-everybody takes care of her and they all love her. Because $I$ come fromabig family. I've had so much help with her.

\section{Friendship Drawback of Hispanic Culture}

Angelica's father, who commented that she had "her little circle of cousins," also went onto reflect on how getting together with the multi-generational family every Sunday afternoon prevents time and energy for connecting with friends and neighbors. He stated, "I think that's one of our values that we have that I think might get in the way of just letting the kids out the front door and go play in the neighborhood." Thus, for many Hispanic families the cousins and other relatives provide a ready context for friendships to flourish, yet there can be "opportunity costs" for establishing friends who are not relatives.

Two of the parents of the younger females particularly stressed that their cultural values encourage them to be overprotective of their daughters as contrasted to Euro-American child-rearing practices. Phyllis's mother stated that her tendency to be overprotective caused her to keep her daughter in the house or in the yard and not allow her to cross the street to play with a friend or especially to go down the street several houses to play with neighborhood children. "I'm talking about my family, we are very overprotective, strict, more strict I think with our children."

\section{Discussion}

In our discussion we focus on (a) friendship criteria, (b) friendship support model, and (c) the role of Hispanic culture.

\section{Friendship Criteria}

We gained insight in this study about our original criteria that the friend should be someone without a disability whose age is within 18 months of the child with a disability. We discuss our unexpected findings, which are an important component in grounded research, related to (a) presence of a disability, (b) proximity in age, and (c) relatives as a ready-made friendship network.

\section{Presence of a Disability.}

There is an emphasis, particularly in the severe disability literature, that it is desirable for children with severe disabilities to have friends who do not have a disability (Grenot-Scheyer, 1994; Kishi \& Meyer, 1994; Salisbury et al., 1995; Staub, 1998). This trend is consistent with inclusive education, whose proponents emphasize social relationships among children and youth with disabilities and their classmates who do not have disabilities. In defining friendship, we were particularly interested in studying friendships of children with a disability and their friends who do not have a disability.

Four of the friendship sets did not meet this criteria (much to our chagrin because we had understood in advance that they did meet the criteria). In one instance, the friend had a mild speech impairment; and in three instances the friend had been identified as having a learning disability (in one of these situations, the student also had a health impairment). The disabilities had been invisible to the community research site coordinator. Furthermore, the presence 
of these disabilities was a non-issue within the friendship. In one friendship set, the friend who had been identified as not having a disability revealed during the interview that in the week intervening between initially agreeing to participate and the interview taking place, she had been identified as having a learning disability. This experience caused us to reflect on the changing status of children (i.e., not identified as having a disabil-ity last week but identified as having a disability this week) and the illusiveness of the criteria of not having a disability, in some situations.

It was obvious to us that whether or not someone has a disability did not appear nearly as much to be an issue for children and youth as it was for $u s$ and other professionals in the field of severe disability who advocate for inclusive education. We believe that this issue warrants further investigation to ensure that the adultinitiated facilitation of friendships for children with disabilities does not unintentionally stigmatize potential friends who happen to have a disability. Perhaps, in an effort to ensure that "all children belong" in inclusive classrooms, there may be unintended negative consequences of discouraging friendships between two children, both of whom have a disability. Interestingly, in the stakeholder review, the one adult with a disability who participated (who also is a strong advocate for inclusive education) identified this point as the basis of what she liked best about the article. She commented: "[this point] is so true-we could do a whole article on that issue."

Proximity in age. A permeating theme of the literature on inclusive education is the importance of age-appropriate placement (Sailor, 1991). After strongly emphasizing the importance of the age criteria to the research site coordinator in Hartford, we were surprised to learn, when Angel and Daniel arrived for the interview, that they differed in age by 5 years. Rather than disqualify them, we proceeded with the interview. Perhaps of all of the friendships we explored, it was this one that had the genuine chemistry of connection.

In terms of support, Daniel and Angel gave each other more emotional support than any of the other friendship sets. Also, there was more instrumental support coming from Angel (a youth with a disability) to Daniel (friend without a disability) than in any other friendship. They were the only ones we interviewed that gave future projections of their friendship that extended into adulthood.
Learning about the incredible bond that these two young people had with each other causes us to be interested in future research on the benefits and drawbacks of cross-age friendships versus same-age friendships and to be not only open, but also eager, to explore a broader range of friendship possibilities.

Relatives as friends. Although not stated in our criteria, it had not occurred to us that the friend might be a relative (i.e., cousin). If the fact that the children might be related had occurred to us, we probably would have added another criteria stating that the children would not be relatives. Perhaps it is a permeating EuroAmerican perspective about friendship to assume that friends would be unrelated. Our beliefs were enriched and broadened by considering the possibility for friendships that exist in extended family networks. We address this point more completely later in this section when we discuss the cultural strengths and resources that these Hispanic children brought to their friendships.

\section{Friendship Support Conceptual Framework}

The friendship support conceptual framework (Figure 1) has potential heuristic value in guiding research and facilitation related to friendships. In terms of research, we believe that a useful next step would be for Participatory Action Research teams (Meyer et al., 1998; Santelli, Singer, DiVenere, Ginsberg, \& Powers, 1998; Turnbull et al., 1998) to be used as an approach to further study the nature and depth of friendship. In such an approach, researchers, children with disabilities, children without disabilities, parents, and teachers might work together to facilitate friendships on a longitudinal basis and to document both the facilitation process and outcomes. Facilitation might concentrate on depth (i.e., children with a disability starting at the level of having acquaintances; moving from the level of acquaintances to casual friendships; and finally moving from casual friendships to intimate friendships). This friendship development might be enhanced across relationship domains (i.e., the nature and extent of companionship, instrumental support, and emotional support) that is reciprocally provided. Hays' (1984) research suggests that successful friendship sets have particular patterns both in the nature of the support provided and in the timing of support leading to a level of intimacy as contrasted to relationships that do not progress in friendship intensity. His work of 
longitudinally and proactively following relationships provides a model for the research we suggest for the disability field, with the addition of 'intervention aimed at the facilitation of shared companionship and reciprocal instrumental and emotional support.

One facilitation application of Hays' (1984) work might be to have Making Action Plans (MAPS) and other personal futures planning groups (Falvey, Forest, Pearpoint, \& Rosenberg, 1994; Mount \& Zwernik, 1988; Smull \& Harrison, 1992; Turnbull \& Turnbull, 1996) creatively brainstorm to identify what the child with a disability might give and what that child might receive to enhance companionship, instrumental support, and emotional support, as illustrated in Table 2. These recommendations would need to be based on the child's strengths, preferences, interests, and resources. After developing a plan for types of support that might be exchanged, the group could develop a facilitation plan outlining the supports, resources, and instruction that would be provided to the child with a disability and the friend in being able to fully actualize the experience of reciprocal friendship. other papers, we focus on the role of parents (Turnbull, Pereira, \& BlueBanning, 1999) and teachers (Turnbull, Pereira, \& Blue-Banning, 1999) as friendship facilitators based on other data collected in this same study. Ultimately, our vision is that children with disabilities will have opportunities to increase the quantity and quality of friendships with a wide range of others, including those with and without disability, those at similar and different ages, and those who are relatives and non-relatives.

\section{Role of Hispanic Culture}

A key Hispanic value is familism, which emphasizes the interdependence of extended families (Anderson, 1989; Zuniga, 1998). Familism results in a network of support, sense of belonging, and emotional security that comes from the extended family. Given the importance of a strengths orientation to cultural diversity, special educators can recognize the relatives of many Hispanic families as being valuable contributors to the quantity and quality of friendships (Harry, 1998).

Some of the families and teachers in this study emphasized the benefit of having a broad

Table 2

Enhancement of Friendship Support for Meili

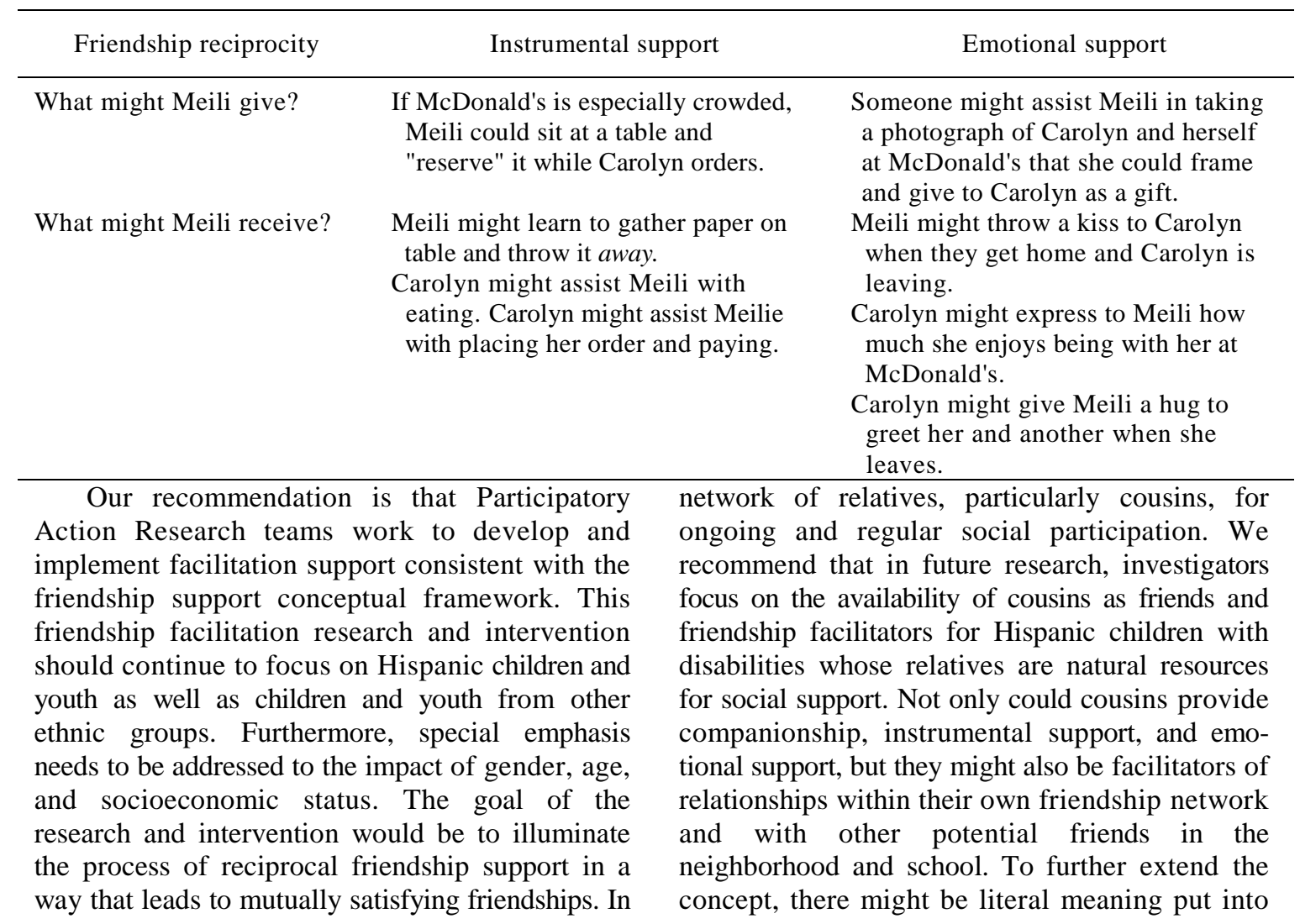


Angelica's father's comment that she had "her little circle of cousins." Although he was not using this phrase literally to imply the structure for friendship facilitation that is present in the circle of friends approach (Falvey et al., 1994), a circle of cousins might be a valuable variation of the circle of friends approach.

During the member check, a mother pointed out the struggle of reducing time spent with family to promote friendship with peers. Furthermore, she indicated that Hispanics are interested in having friends, which she expressed is contrary to the stereotypical images that some Euro-American professionals have. Because of the intensity of interactions with the relatives, she explained that some Hispanics may be less proactive in initiating friendships in settings such as school.

Several parents discussed overprotective-ness as a barrier that prevented them from giving their children sufficient freedom to explore friendships (in the situations of the families in this study, overprotectiveness was not raised as an issue in ensuring the safety of children in neighborhoods where there are potential and actual threats to their safety. We acknowledge that in some situations what several parents and professionals might perceive to be overprotectiveness may involve necessary safety precautions). At least for one family in this study, overprotectiveness served as potential cultural strength. Angelica's father described himself as overprotective, with the result being that he often accompanied his daughters to parties and community events. The result of his accompaniment is that he served as a facilitator in these settings. For example, he went along with Angelica to catechism classes and supported the teacher in knowing how to appropriately include her. Thus, a potentially useful area of inquiry would be to investigate how protective, even overprotective parental strategies, could actually be turned into parental friendship and inclusion facilitation. Another important area of future inquiry, as recommended through the member check, is investigating the impact of the level of acculturation and the geographical location of the family in terms of parental interest in friendship and inclusion facilitation.

\section{Conclusion: Toward Understanding Friendships}

We particularly want to underscore the emphasis in the friendships on companionship and emotional support as contrasted to instrumental support, indicating that these friendships were not solely about "help." The nature of the friendships enabled us to revise our own values and become more informed through appreciating and respecting Hispanic culture and individual choices. Finally, consistent with a Participatory Action Research approach, we particularly appreciate the guidance of the Participatory Action Research committee in focusing solely on Hispanic children and their families. By having a more intense cultural immersion, we believe we profited from the strengths and resources related to personal relationships that the Hispanic culture has to share with other cultures.

We hope that the friendship support conceptual framework (Figure 1) provided in this study will be useful to the field in simultaneously considering relationship domains, the extent of reciprocity within relationship domains, and the depth of friendship. By conceptualizing an approach for friendship facilitation from the earliest years, we hope that there will be a lifespan emphasis on enhancing friendships as a key aspect of increasing quality of life for individuals with disabilities and their families (Schalock, 1997).

"Of all the things that wisdom provides to make life entirely happy, much the greatest is the possession of friendship." -Epicurus

\section{References}

Anderson, $P$. P. (1989). Issues in serving culturally diverse families of young children with disabilities. Early Childhood Development and Care, 50,167-188.

Antonucci, T. C., \& Jackson, J. S. (1990). The role of reciprocity in social support. In B. R. Sarason, I. 0. Sarason, \& 0. R. Pierce (Eds.), Social support: An interactional view (pp. 173-198). New York: Wiley.

Bandura, A. (1982): Self-efficacy mechanism in human agency. American Psychologist, 37, 122-147.

Belle, D. (Ed.). (1989). Children's social networks and social supports. New York: Wiley.

Berg, B. L. (1998). Qualitative research methods for the socal sciences (3rd ed). Needham Heights, MA: Allyn \& Bacon.

Berndt, T. J. (1989). Obtaining support from friends during childhood and adolescence. In D. Belle (Ed.), Children's social networks and social supports (pp. 308-33 1). New York: Wiley.

Cobb, S. (1979). Social support and health through the life course. In M. W. Riley (Ed.), Aging from birth to death: Interdisciplinary perspectives (pp. 93-106). Boulder, CO: Westview Press.

Creswell, J. W. (1994). Research design: Qualitative and quantitative approaches. Thousand Oaks, CA: Sage.

Cutrona, C. E., \& Russell, D. W. (1990). Type of social support and specific stress: Toward a theory of 
optimal matching. In B. R. Sarason, L. G. Sarason, \& 0. R. Pierce (Eds.), Social support: An interactional view (pp. 319-366). New York: Wiley.

Evans, I. M., Salisbury, C. L., Palombaro, M. M., Berryman, J., \& Hollowood, T. M. (1992). Peer interactions and social acceptance of elementary-age children with severe disabilities in an inclusive school. Journal of the Association for Persons with Severe Handicaps, 17, 205-212.

Falvey, M. A., Forest, M., Pearpoint, J., \& Rosenberg, R. L. (1994). Building connections. In J. S. Thousand, R. A. Villa, \& A. I. Nevin (Eds.), Creativity and collaborative learning: A practical guide for empowering students and teachers (pp. 347-368). Baltimore: Brookes.

Farmer, T. W., \& Farmer, E. M. Z. (1996). Social relationships of students with exceptionalities in mainstream classrooms: Social networks and homophily. Exceptional Children, 62, 431-450.

Feiring, C., \& Lewis, M. (1989). The social networks of girls and boys from early through middle childhood. In D. Belle (Ed.), Children's social networks and social supports (pp. 119-150). New York: Wiley.

Gallegos, J. S. (1991). Culturally relevant services for Hispanic elderly. In M. Sotomayor (Ed.), Empowering Hipanic families: A critical issue for the '90s (pp. 173190). Milwaukee: Family Service America.

Grenot-Scheyer, M. (1994). The nature of interactions between students with severe disabilities and their friends and acquaintances without disabilities. Journal of the Association for Persons with Severe Handicaps, 19, 253-262.

Grenot-Scheyer, M., Staub, D., Peck, C. A., \& Schwartz, I. S. (1998). Reciprocity and friendships: Listening to the voices of children and youth with and without disabilities. In L. H. Meyer, H. S. Park, M. Grenot-Scheyer, I. S. Schwartz, \& B. Harry (Eds.), Making friends: The influences of culture and development (pp. 149167). Baltimore: Brookes.

Gutiérrez, L. M. (1995). Empowerment and Latinos: Implications for practice. Family Resource Coalition Report, 13(3 \& 4), 5-8.

Harry, B. (1992). Cultural diversity, families, and the special education system. New York: Teachers College Press.

Harry, B. (1998). Parental visions of una vida normal/a normal life: Cultural variations on a theme. In L. H. Meyer, H. S., Park, M. Grenot-Scheyer, I. S. Schwartz, \& B. Harry (Eds.), Making friends: The influences of culture and development (pp. 47-62). Baltimore: Brookes.

Hays, R. B. (1984). The development and maintenance of friendship. Journal of Social and Personal Relationships, 1, 75-98.

Helmstetter, E., Peck, C. A., \& Giangreco, M. F. (1994). Outcomes of interactions with peers with moderate or severe disabilities: A statewide survey of high school students. Journal of the Association for Persons with Severe Disabilities, 19, 263-276.

Hoshmand, L. T, \& Polkinghorne, D. E. (1992). Redefining the science-practice relationship and professional training. American Psychologist, 47, 55-66.

Kennedy, C. H., Horner, R. H., \& Newton, J. S. (1990). The social networks and activity patterns of adults with severe disabilities: A correlational analysis. Journal of the Association for Persons with Severe Handicaps, 15, 86-90.

Kennedy, C. H., \& Itkonen, T (1996). Social relationships, influential variables, and change across the life span. In L. K. Koegel, R. L. Koegel, \& 0. Dunlap (Eds.), Positive behavioral support: Including people with difficult behavior in the community ( $\mathrm{pp}$. 287-304). Baltimore: Brookes.

Kishi, 0. S., \& Meyer, L. H. (1994). What children report and remember: A six-year follow-up of the effects of social contact between peers with and without severe disabilities. Journal of the Association for Persons with Severe Handicaps, 19, 277-289.

Krueger, R. A. (1998). Developing questions for focus groups. Thousand Oaks, CA: Sage.

Lather, P. (1986). Research as praxis. Harvard Educational Review, 56, 257-277.

Mann, G., \& Marín, B. V. (1991). Research with Hispanic populations. Newbury Park, CA: Sage.

Merriam, S. B. (1998). Qualitative research and case study applications in education. San Francisco: Jossey-Bass.

Meyer, L. H., Park, H. S., Grenot-Scheyer, M., Schwartz, I., \& Harry, B. (1998). Participatory research approaches for the study of the social relationships of children and youth. In L. H. Meyer, H. S. Park, I. Schwartz, M. Grenot-Scheyer, \& B. Harry (Eds.), Understanding the social Lives of children and youth with diverse abilities: The role of culture and development. Baltimore: Brookes.

Mount, B., \& Zwernik, K. (1988). It's never too early, it's never too late: A booklet about personal planning for persons with developmental disabilities, their families and friends, case managers, service providers, and advocates. St. Paul, MN: Metropolitan Council.

Patton, M. Q. (1990). Qualitative evaluation and research methods (2nd ed.). Newbury Park, CA: Sage.

Reid, M., Landesman, S., Treder, R., \& Jaccard, J. (1989). My family and friends: Six to twelve-year-old children's perceptions of social support. Child Development, 60, 896-910.

Ross, H. S., Cheyne, J. A., \& Lollis, S. P. (1988). Defining and studying reciprocity in young children. In S. W. Duck (Ed.), Handbook of personal relationships (pp. 143-160). New York: Wiley.

Sailor, W. (1991). Special education in the restructured school. Remedial and Special Education, $12(6), 8-22$.

Salisbury, C. L., Gallucci, C., Palombaro, M. M., \& Peck, C. A. (1995). Strategies that promote social relations among elementary students with and without severe disabilities in inclusive schools. Exceptional Children, 62, 125-137.

Salisbury, C. L., \& Palombara, M. M. (1998). Friends and acquaintances: Evolving relationships in an inclusive elementary school. In L. H. Meyer, H. S. Park, M. Grenot-Scheyer, L. S. Schwartz, \& B. Harry (Eds.), Making friends: The influences of culture and development (pp. 65-80). Baltimore: Brookes.

Santelli, B., Singer, G. H. S., DiVenere, N., Ginsberg, C., \& Powers, L. (1998). Participatory action research: Reflections on critical incidents in a Participatory Action Research project. Journal of the Association for Persons with Severe Handicaps, 23, 211-222. 
Sarason, B. R., Sarason, I. G., \& Pierce, G. R. (Eds.). (1990). Social support: An interactional view. New York: Wiley.

Schaefer, C., Coyne, J. C., \& Lazarus, R. S. (1981). The health-related functions of social support. Journal of Behavioral Medicine, 4, 381-406.

Seidel, J., Friese, S., \& Leonard, D. C. (1995). The ethnograph V4.0: A user's guide. Salt Lake City, UT Quality Research Associates.

Smull, M., \& Harrison, S. B. (1992). Supporting people with severe reputations in the community. Alexandria, VA: National Association of State Mental Retardation Program Directors.

Snell, M. E., \& Vogtle, L. K. (1997). Facilitating relationships of children with mental retardation in schools. In R. L. Schalock (Ed.), Quality of life: Application to persons with disabilities (Vol. II, pp. 43-62). Washington, DC: American Association on Mental Retardation.

Soriano, F. I. (1991). U.S. Hispanics and their famlies: A sociocultural portrait. Paper prepared for the International Conference on the Unity of Sciences, Seoul, Korea.

Staub, D. (1998). Delicate threads: Friendships between children with and without special needs in inclusive settings. Bethesda: Woodbine House.

Staub, D., Schwartz, 1. S., Gallucci, C., \& Peck, C. A. (1994). Four portraits of friendship at an inclusive school. Journal of the Association for Persons with Severe Handicaps, 19, 314-325.

Sullivan, H. S. (1953). The interpersonal theory of psychiatry. New York: Norton.

Taylor, S. J., \& Bogdan, R. (1998). Introduction to qualitative research methods: A guidebook and resource (3rd ed.). New York: Wiley.

Turnbull, A. P., Friesen, B. J., \& Ramirez, C. (1998). Participatory action research as a model of conducting ducting family research. Journal of the Association for Persons with Severe Handicaps, 23, 178-188.
Turnbull, A. P., Pereira, L., \& Blue-Banning, M. J. (1999). Parents' facilitation of friendships between their children with a disability and friends without a disability. Journal of the Association for Persons with Severe Handicaps, 24, 85-99.

Turnbull, A. P., Pereira, L., \& Blue-Banning, M. J. (in press). Teachers as friendships facilitators. Teaching Exceptional Children.

Turnbull, A. P., \& Turnbull, H. R. (1996). Group Action Planning as a strategy for providing comprehensive family support. In R. L. Koegel, L. K. Koegel, \& G. Dunlap (Eds.), Positive behavioral support: Including people with difficult behavior in the community (pp. 99-114). Baltimore: Brookes.

U.S. Bureau of the Census. (1995). Race and Hispanic origin census profile. Washington, DC: U.S. Department of Commerce, Economics and Statistics Administration.

U.S. Department of Education. (1993, February). 1990 elementary and secondary schools civil rights survey. Adjusted national estimated data. Washington, DC: U.S. Department of Education, Office for Civil Rights.

Van der Kilft, E., \& Kunc, N. (1994). Beyond benevolence: Friendship and the politics of help. In J. S. Thousand, R. A. Villa, \& A. I. Nevin (Eds.), Creativity and collaborative learning: A practical guide for empowering students and teachers ( $\mathrm{pp}$. 391-402). Baltimore: Brookes.

Walker, C. L. (1987). Hispanic achievement: Old views and new perspectives. In H. T. Trueba (Ed.), Success or failure? (pp. 15-32). Cambridge, MA: Newbury House.

Zuniga, M. E. (1998). Families with Latino roots. In E. W. Lynch \& M. J. Hanson (Eds.), Developing cross-cultural competence: A guide for working with children and their families (pp. 209-250). Baltimore: Brookes.

Received 12/14/98, first decision 3/15/99, accepted 5/13/99.

Editor-in-Charge: Steven J. Taylor

We extend special thanks to members of the Participatory Action Research Committee, including Kathy Berg, Glen Dunlap, Glenn Gabbard, Larry Irvin, Michelle Gaskelle, Judy O'Halloran, Carmen Ramfrez, Jane Scarffe, and Nancy Verderber.

Authors: ANN P. TURNBULL, EdD, Co-Director, MARTHA BLUE-BANNING, PhD, Research Associate, Beach Center on Families and Disability, University of Kansas, 3111 Haworth Hall, Lawrence, KS 66045-2930. LOURDES PEREIRA, Adjunct Professor, Georgia Perimeter College, Dunwoody Campus, 2101 Womack Rd., Durwoody, GA 30338.

This research was supported by a grant from the National Institute on Disability and Rehabilitation Research to the Beach Center on Disability, Grant \#H133B30070. 